\title{
HAHN'S PROBLEM WITH RESPECT TO SOME PERTURBATIONS OF THE RAISING OPERATOR $X-c$
}

\author{
Baghdadi Aloui \\ University of Gabes, Higher Institute of Industrial Systems of Gabes \\ Salah Eddine Elayoubi Str., 6033 Gabes, Tunisia \\ Baghdadi.Aloui@fsg.rnu.tn \\ Jihad Souissi \\ University of Gabes, Faculty of Sciences of Gabes \\ Erriadh Str., 6072 Gabes, Tunisia \\ jihadsuissi@gmail.com
}

\begin{abstract}
In this paper, we study the Hahn's problem with respect to some raising operators perturbed of the operator $X-c$, where $c$ is an arbitrary complex number. More precisely, the two following characterizations hold: up to a normalization, the $q$-Hermite (resp. Charlier) polynomial is the only $H_{\alpha, q}$-classical (resp. $\mathcal{S}_{\lambda}$-classical) orthogonal polynomial, where $H_{\alpha, q}:=X+\alpha H_{q}$ and $\mathcal{S}_{\lambda}:=(X+1)-\lambda \tau_{-1}$.
\end{abstract}

Keywords: Orthogonal polynomials, Linear functional, $\mathcal{O}$-classical polynomials, Raising operators, $q$-Hermite polynomials, Charlier polynomials.

\section{Introduction}

Let $\mathcal{O}$ be a linear operator acting on the space of polynomials which sends polynomials of degree $n$ to polynomials of degree $n+n_{0}$, where $n_{0}$ is a fixed integer $\left(n \geq 0\right.$ if $n_{0} \geq 0$ and $n \geq\left|n_{0}\right|$ if $\left.n_{0}<0\right)$. We call a sequence $\left\{P_{n}\right\}_{n \geq 0}$ of orthogonal polynomials $\mathcal{O}$-classical if $\left\{\mathcal{O} P_{n}\right\}_{n \geq 0}$ is also orthogonal.

In particular, if $\mathcal{O}=D$, the standard derivative, we recover the know family of classical orthogonal polynomials (Hermite, Laguerre, Bessel and Jacobi). This characterization is called Hahn's characterization (see $[11,18]$ ) of the classical orthogonal polynomials. If $\mathcal{O}=H_{q}$, where

$$
H_{q} f(x)=\frac{h_{q} f(x)-f(x)}{(q-1) x}, \quad q \neq 1, \quad h_{q} f(x)=f(q x),
$$

we recover the so-called $H_{q}$-classical polynomials (for more details, see [12]). We can also cite [14], where the authors described the all $D_{\omega}$-classical orthogonal polynomials, with

$$
D_{\omega} f(x):=\frac{\tau_{-\omega} f(x)-f(x)}{w}, \quad \omega \neq 0, \quad \tau_{-\omega} f(x)=f(x+\omega) .
$$

The literature on these topics is extremely vast. See further examples in $[1-5,7,8,11,12,14]$.

In this paper we consider some raising operators related to the operator $X$. It is easy to see that the orthogonality is not preserved by $X$, then we can consider and study some perturbed operators. Here we consider the following two operators $(c=0$ or $c=1)$ :

$$
\begin{gathered}
H_{\alpha, q}:=X+\alpha H_{q} \\
\mathcal{S}_{\lambda}:=(X+1)-\lambda \tau_{-1},
\end{gathered}
$$


and we study the same problem, called Hahn's problem. More precisely, we find all orthogonal polynomial sequences $\left\{P_{n}\right\}_{n \geq 0}$ such that $\left\{\mathcal{O} P_{n}\right\}_{n \geq 0}, \mathcal{O}=H_{\alpha, q}$ or $\mathcal{S}_{\lambda}$, are also orthogonal. As a result, we conclude that the $q$-Hermite polynomial sequence is the only $H_{\alpha, q}$-classical sequence and the Charlier polynomial sequence is the only $\mathcal{S}_{\lambda}$-classical sequence.

The structure of the paper is the following. In Section 2, a basic background about forms of orthogonal polynomials is given. In Section 3, we show that, up to a dilatation, the $q$-Hermite (resp. Charlier) polynomial is the only $H_{\alpha, q}$-classical (resp. $\mathcal{S}_{\lambda}$-classical) orthogonal polynomial. In Section 4 , we give a conclusion and describe some prospects.

\section{Preliminaries}

Let $\mathbb{P}$ be the linear space of polynomials in one variable with complex coefficients and $\mathbb{P}^{\prime}$ be its dual space, whose elements are forms. We denote by $\langle u, p\rangle$ the action of $u \in \mathbb{P}^{\prime}$ on $p \in \mathbb{P}$. In particular, we denote by $(u)_{n}:=\left\langle u, x^{n}\right\rangle, n \geq 0$, the moments of $u$. Let us define the following operations in $\mathbb{P}^{\prime}$. For any form $u$, any polynomial $f$, and any $(a, b, c) \in \mathbb{C} \backslash\{0\} \times \mathbb{C}^{2}$, let $D u=u^{\prime}, f u$, $(x-c)^{-1} u, \tau_{-b} u$ and $h_{a} u$ be the forms defined by duality, [16]:

$$
\begin{gathered}
\langle f u, p\rangle:=\langle u, f p\rangle, \quad\left\langle u^{\prime}, p\right\rangle:=-\left\langle u, p^{\prime}\right\rangle, \quad(f u)^{\prime}=f^{\prime} u+f u^{\prime}, \\
\left\langle h_{a} u, p\right\rangle:=\langle u, p(a x)\rangle, \quad\left\langle\tau_{-b} u, p\right\rangle:=\langle u, p(x-b)\rangle, \\
\left\langle(x-c)^{-1} u, p\right\rangle:=\left\langle u, \frac{p(x)-p(c)}{x-c}\right\rangle, \quad p \in \mathbb{P} .
\end{gathered}
$$

A form $u$ is called normalized if it satisfies $(u)_{0}=1$. We assume that the forms used in this paper are normalized.

Let $\left\{P_{n}\right\}_{n>0}$ be a sequence of monic polynomials (MPS) with $\operatorname{deg} P_{n}=n$ and let $\left\{u_{n}\right\}_{n>0}$ be its dual sequence, $u_{n} \in \mathbb{P}^{\prime}$, defined by $\left\langle u_{n}, P_{m}\right\rangle=\delta_{n, m}, n, m \geq 0$. Notice that $u_{0}$ is said to be the canonical functional associated with the MPS $\left\{P_{n}\right\}_{n \geq 0}$. The sequence $\left\{P_{n}\right\}_{n \geq 0}$ is called symmetric when $P_{n}(-x)=(-1)^{n} P_{n}(x), n \geq 0$.

Let us recall the following result [17].

Lemma 1. For any $u \in \mathbb{P}^{\prime}$ and any integer $m \geq 1$, the following statements are equivalent:

(i) $\left\langle u, P_{m-1}\right\rangle \neq 0, \quad\left\langle u, \quad P_{n}\right\rangle=0, n \geq m$.

(ii) $\exists \lambda_{\nu} \in \mathbb{C}, \quad 0 \leq \nu \leq m-1, \quad \lambda_{m-1} \neq 0 \quad$ such that $\quad u=\sum_{\nu=0}^{m-1} \lambda_{\nu} u_{\nu}$.

As a consequence, the dual sequence $\left\{u_{n}^{[1]}\right\}_{n \geq 0}$ of $\left\{P_{n}^{[1]}\right\}_{n \geq 0}$ where

$$
P_{n}^{[1]}(x):=(n+1)^{-1} P_{n+1}^{\prime}(x), \quad n \geq 0,
$$

is given by

$$
D u_{n}^{[1]}=-(n+1) u_{n+1}, \quad n \geq 0 .
$$

Similarly, the dual sequence $\left\{\tilde{u}_{n}\right\}_{n \geq 0}$ of $\left\{\tilde{P}_{n}\right\}_{n \geq 0}$, where

$$
\tilde{P}_{n}(x):=a^{-n} P_{n}(a x+b)
$$

with $(a, b) \in \mathbb{C} \backslash\{0\} \times \mathbb{C}$, is given by

$$
\tilde{u}_{n}=a^{n}\left(h_{a^{-1}} \circ \tau_{-b}\right) u_{n}, n \geq 0 .
$$

The form $u$ is called regular if we can associate with it a sequence $\left\{P_{n}\right\}_{n \geq 0}$ such that

$$
\left\langle u, P_{n} P_{m}\right\rangle=r_{n} \delta_{n, m}, \quad n, m \geq 0, \quad r_{n} \neq 0, \quad n \geq 0 .
$$


The sequence $\left\{P_{n}\right\}_{n \geq 0}$ is then called a monic orthogonal polynomial sequence (MOPS) with respect to $u$. Note that $u=(u)_{0} u_{0}$, with $(u)_{0} \neq 0$. When $u$ is regular, let $F$ be a polynomial such that $F u=0$. Then $F=0,[16]$.

Proposition 1 [16]. Let $\left\{P_{n}\right\}_{n \geq 0}$ be a MPS with $\operatorname{deg} P_{n}=n, n \geq 0$, and let $\left\{u_{n}\right\}_{n \geq 0}$ be its dual sequence. The following statements are equivalent.

(i) $\left\{P_{n}\right\}_{n \geq 0}$ is orthogonal with respect to $u_{0}$.

(ii) $u_{n}=\left\langle u_{0}, P_{n}^{2}\right\rangle^{-1} P_{n} u_{0}, \quad n \geq 0$.

(iii) $\left\{P_{n}\right\}_{n \geq 0}$ satisfies the three-term recurrence relation

$$
\left\{\begin{array}{l}
P_{0}(x)=1, \quad P_{1}(x)=x-\beta_{0}, \\
P_{n+2}(x)=\left(x-\beta_{n+1}\right) P_{n+1}(x)-\gamma_{n+1} P_{n}(x), \quad n \geq 0,
\end{array}\right.
$$

where $\beta_{n}=\left\langle u_{0}, x P_{n}^{2}\right\rangle\left\langle u_{0}, P_{n}^{2}\right\rangle^{-1}, n \geq 0$ and $\gamma_{n+1}=\left\langle u_{0}, P_{n+1}^{2}\right\rangle\left\langle u_{0}, P_{n}^{2}\right\rangle^{-1} \neq 0, \quad n \geq 0$.

If $\left\{P_{n}\right\}_{n \geq 0}$ is a MOPS with respect to the regular form $u_{0}$, then $\left\{\tilde{P}_{n}\right\}_{n \geq 0}$ is a MOPS with respect to the regular form $\tilde{u}_{0}=\left(h_{a^{-1}} \circ \tau_{-b}\right) u_{0}$, and satisfies [15]

$$
\left\{\begin{array}{l}
\tilde{P}_{0}(x)=1, \quad \tilde{P}_{1}(x)=x-\tilde{\beta}_{0}, \\
\tilde{P}_{n+2}(x)=\left(x-\tilde{\beta}_{n+1}\right) \tilde{P}_{n+1}(x)-\tilde{\gamma}_{n+1} \tilde{P}_{n}(x), \quad n \geq 0,
\end{array}\right.
$$

where $\tilde{\beta}_{n}=a^{-1}\left(\beta_{n}-b\right)$ and $\tilde{\gamma}_{n+1}=a^{-2} \gamma_{n+1}$.

A MOPS $\left\{p_{n}\right\}_{n \geq 0}$ is called $D$-classical, if $\left\{D p_{n}\right\}_{n \geq 0}$ is also orthogonal (Hermite, Laguerre, Bessel or Jacobi), [10,11]. Moreover, if $\left\{p_{n}\right\}_{n \geq 0}$ is orthogonal with respect to $u_{0}$, then there exists a monic polynomial $\phi$ with $\operatorname{deg} \phi \leq 2$ and a polynomial $\psi$ with $\operatorname{deg} \psi=1$ such that $u_{0}$ satisfies a Pearson's equation (PE) [15]

$$
D\left(\phi u_{0}\right)+\psi u_{0}=0 .
$$

Any shift leaves invariant the $D$-classical character. Indeed, the shifted linear functional $\tilde{u}=$ $\left(h_{a^{-1}} \circ \tau_{-b}\right) u$ fulfills the equation

$$
(\widetilde{\Phi} \tilde{u})^{\prime}+\widetilde{\Psi} \tilde{u}=0
$$

where (see $[15,16])$

$$
\widetilde{\Phi}(x)=a^{-t} \Phi(a x+b) \quad \text { and } \quad \widetilde{\Psi}(x)=a^{1-t} \Psi(a x+b) .
$$

\section{Hahn's problem with respect to some perturbations of the raising operator $X-c$}

Clearly, the orthogonality is not preserved by the operator $X-c$, which is given by

$$
(X-c)(f(x))=(x-c) f(x), \quad f \in \mathbb{P} .
$$

Our goal, in this section is to describe all $\mathcal{O}$-classical orthogonal polynomials. More precisely, we find all orthogonal polynomial sequences $\left\{P_{n}\right\}_{n \geq 0}$ such that $\left\{\mathcal{O} P_{n}\right\}_{n \geq 0}$ are also orthogonal, where $\mathcal{O}=H_{\alpha, q}$ or $\mathcal{O}=\mathcal{S}_{\lambda}$ are the operators defined by (1.1) and (1.2). This operators are two perturbations of the operator $X-c$ where $c=0$ and $c=1$. 


\subsection{Orthogonal polynomials via raising operator $X-\alpha H_{q}$}

Let us introduce the following lemma.

Lemma 2 [12]. The following properties hold

$$
\begin{aligned}
H_{q}(f g)(x)= & f(x)\left(H_{q} g\right)(x)+g(x)\left(H_{q} f\right)(x)+(q-1) x\left(H_{q} f\right)(x)\left(H_{q} g\right)(x), \quad f, g \in \mathcal{P}, \\
& H_{q}(f u)=\left(h_{q^{-1}} f\right) H_{q} u+q^{-1}\left(H_{q^{-1}} f\right) u, \quad f \in \mathcal{P}, \quad u \in \mathcal{P}^{\prime} .
\end{aligned}
$$

where

$$
H_{q} f(x)=\frac{h_{q} f(x)-f(x)}{(q-1) x}, \quad q \neq 1 \quad \text { and } \quad h_{q} f(x)=f(q x) .
$$

Now, recall the operator

$$
\begin{aligned}
H_{\alpha, q}: \mathbb{P} & \longrightarrow \mathbb{P} \\
f & \longmapsto H_{\alpha, q}(f):=x f+\alpha H_{q}(f) .
\end{aligned}
$$

Definition 1. We call a sequence $\left\{P_{n}\right\}_{n \geq 0}$ of orthogonal polynomials $H_{\alpha, q}$-classical if there exists a sequence $\left\{Q_{n}\right\}_{n \geq 0}$ of orthogonal polynomials such that $H_{\alpha, q} P_{n}=Q_{n+1}, n \geq 0$.

For any MPS $\left\{P_{n}\right\}_{n \geq 0}$ we define the MPS $\left\{Q_{n}\right\}_{n \geq 0}$, given by

$$
Q_{n+1}(x):=H_{\alpha, q} P_{n}(x), n \geq 0,
$$

or equivalently

$$
Q_{n+1}(x):=x P_{n}(x)+\alpha\left(H_{q} P_{n}\right)(x), \quad n \geq 0,
$$

with initial value $Q_{0}(x)=1$.

Our next goal is to describe all the $H_{\alpha, q}$-classical polynomial sequences. Note that, we need $\alpha \neq 0$ to ensure that $\left\{Q_{n}\right\}_{n \geq 0}$ is an orthogonal sequence. Indeed, if we suppose that $\alpha=0$, the relation (3.1) becomes, for $x=0, Q_{n+1}(0)=0, n \geq 0$, which contradicts the orthogonality of $\left\{Q_{n}\right\}_{n \geq 0}$.

Clearly, the operator $H_{\alpha, q}$ raises the degree of any polynomial. Such operator is called raising operator $[9,13,19]$. By transposition of the operator $H_{\alpha, q}$, we get

$$
{ }^{t} H_{\alpha, q}=X-\alpha H_{q} .
$$

Denote by $\left\{u_{n}\right\}_{n \geq 0}$ and $\left\{v_{n}\right\}_{n \geq 0}$ the dual basis in $\mathbb{P}^{\prime}$ corresponding to $\left\{P_{n}\right\}_{n \geq 0}$ and $\left\{Q_{n}\right\}_{n \geq 0}$, respectively. Then, according to Lemma 1 and (3.2), the relation

$$
x v_{n+1}-\alpha H_{q}\left(v_{n+1}\right)=u_{n}, \quad n \geq 0,
$$

holds. Assume that $\left\{P_{n}\right\}_{n \geq 0}$ and $\left\{Q_{n}\right\}_{n \geq 0}$ are MOPS satisfying

$$
\begin{aligned}
& \left\{\begin{array}{l}
P_{0}(x)=1, \quad P_{1}(x)=x-\beta_{0}, \\
P_{n+2}(x)=\left(x-\beta_{n+1}\right) P_{n+1}(x)-\gamma_{n+1} P_{n}(x), \quad \gamma_{n+1} \neq 0, \quad n \geq 0,
\end{array}\right. \\
& \left\{\begin{array}{l}
Q_{0}(x)=1, Q_{1}(x)=x-\rho_{0}, \\
Q_{n+2}(x)=\left(x-\rho_{n+1}\right) Q_{n+1}(x)-\varrho_{n+1} Q_{n}(x), \quad \varrho_{n+1} \neq 0, \quad n \geq 0 .
\end{array}\right.
\end{aligned}
$$

Next, a first result will be deduced as a consequence of the relations (3.1), (3.4) and (3.5). 
Proposition 2. The sequences $\left\{P_{n}\right\}_{n \geq 0}$ and $\left\{Q_{n}\right\}_{n \geq 0}$ satisfy the following finite type relation

$$
P_{n}(x)+(q-1) x H_{q}\left(P_{n}\right)(x)=q^{n} Q_{n}(x), \quad n \geq 0 .
$$

P r o o f. Using (3.4), we obtain

$$
H_{q}\left(P_{n+2}\right)(x)=H_{q}\left(\left(x-\beta_{n+1}\right) P_{n+1}\right)(x)-\gamma_{n+1} H_{q}\left(P_{n}\right)(x), \quad n \geq 0 .
$$

According to the Lemma 2, we obtain for $n \geq 0$

$$
H_{q}\left(P_{n+2}\right)(x)=\left(x-\beta_{n+1}\right) H_{q}\left(P_{n+1}\right)(x)+P_{n+1}(x)+(q-1) x H_{q}\left(P_{n+1}\right)(x)-\gamma_{n+1} H_{q}\left(P_{n}\right)(x),
$$

or equivalently

$$
x P_{n+2}(x)+\alpha\left(H_{q} P_{n+2}\right)(x)=Q_{n+3}(x), \quad n \geq 0,
$$

which gives us for $n \geq 0$

$$
\left(x-\beta_{n+1}\right) x P_{n+1}(x)+\alpha\left(q x-\beta_{n+1}\right)\left(H_{q} P_{n+1}\right)(x)-\gamma_{n+1}\left(x P_{n}(x)+\alpha\left(H_{q} P_{n}\right)(x)\right)+\alpha P_{n+1}(x)=Q_{n+3}(x) .
$$

We use (3.1) and the last equation becomes for $n \geq 0$

$$
\left(x-\beta_{n+1}\right) Q_{n+2}(x)+\alpha(q-1) x\left(H_{q} P_{n+1}\right)(x)-\gamma_{n+1} Q_{n+1}(x)+\alpha P_{n+1}(x)=Q_{n+3}(x) .
$$

Inserting (3.5) in (3.6), we obtain

$$
\alpha P_{n+1}(x)+\alpha(q-1) x\left(H_{q} P_{n+1}\right)(x)=\left(\beta_{n+1}-\rho_{n+2}\right) Q_{n+2}(x)+\left(\gamma_{n+1}-\varrho_{n+2}\right) Q_{n+1}(x), \quad n \geq 0 .
$$

In fact, this result is valid for $n+1$ replaced by $n$. More precisely, we have for all $n \geq 0$

$$
\alpha P_{n}(x)+\alpha(q-1) x\left(H_{q} P_{n}\right)(x)=\left(\beta_{n}-\rho_{n+1}\right) Q_{n+1}(x)+\left(\gamma_{n}-\varrho_{n+1}\right) Q_{n}(x),
$$

with the convention $\gamma_{0}=0$. By comparing the degrees in the previous equation, we get $\beta_{n}=\rho_{n+1}, n \geq 0$ and $\alpha q^{n}=\gamma_{n}-\varrho_{n+1}, n \geq 0$. Hence the desired result is proven.

Note that, for $n=0$ the relation (3.1) gives $\rho_{0}=0$, for $n=1$ the Proposition 2 gives

$$
\left(x-\beta_{0}\right)+(q-1) x=q x-\rho_{0}=q x,
$$

then $\beta_{0}=\rho_{1}=0$. Now we establish, in the next lemma, an algebraic relation between the forms $u_{0}$ and $v_{0}$.

Lemma 3. The forms $u_{0}$ and $v_{0}$ satisfy the following relation

$$
v_{0}-(q-1) H_{q}\left(x v_{0}\right)=u_{0} .
$$

P r o o f. According to Proposition 2 we obtain

$$
\left\langle v_{0}-(q-1) H_{q}\left(x v_{0}\right), P_{n}\right\rangle=0, \quad n \geq 1 .
$$

On the other hand,

$$
\left\langle v_{0}-(q-1) H_{q}\left(x v_{0}\right), P_{0}\right\rangle=1,
$$

since $\left\{Q_{n}\right\}_{n \geq 0}$ is orthogonal with respect to the form $v_{0}$, where $v_{0}$ is supposed normalized. According to Lemma 1 and using (3.8), we obtain the desired result.

Based on the last lemma, we can state the following theorem. 
Theorem 1. The form $v_{0}$ satisfies the following Pearson's equation

$$
\left(H_{q} v_{0}\right)-\frac{1}{\alpha} x v_{0}=0
$$

and then the scaled $q$-Hermite polynomial sequence is the only $H_{\alpha, q^{-}}$classical sequence.

P r o o f. According to Proposition 1 (ii), the relation (3.3) can be written as follows

$$
x Q_{n+1}(x) v_{0}-\alpha H_{q}\left(Q_{n+1} v_{0}\right)=\lambda_{n} P_{n}(x) u_{0}, \quad n \geq 0,
$$

where

$$
\lambda_{n}:=\left\langle v_{0}, Q_{n+1}^{2}\right\rangle\left\langle u_{0}, P_{n}^{2}\right\rangle^{-1}, \quad n \geq 0 .
$$

Making $n=0$ in (3.10), we get

$$
x^{2} v_{0}-\alpha H_{q}\left(x v_{0}\right)=-\alpha u_{0}, \quad\left(Q_{1}(x)=x, \quad \varrho_{1}=-\alpha\right) .
$$

Substituting this relation in (3.7), we obtain

$$
q H_{q}\left(x v_{0}\right)-\frac{1}{\alpha}\left(x^{2}+\alpha\right) v_{0}=0 .
$$

Note that we have $q H_{q}\left(x v_{0}\right)=x\left(H_{q} v_{0}\right)+v_{0}$, then

$$
\left(H_{q} v_{0}\right)-\frac{1}{\alpha} x v_{0}=0
$$

which gives

$$
\left(\left(H_{q} v_{0}\right)-\frac{1}{\alpha} x v_{0}\right)_{n+1}=0, \quad n \geq 0,
$$

and then

$$
\left(v_{0}\right)_{n+2}=-\alpha[n]_{q}\left(v_{0}\right)_{n}, \quad n \geq 0 .
$$

Moreover, $\left(v_{0}\right)_{1}=\rho_{1}=0$, hence $\left(v_{0}\right)_{2 n+1}=0, n \geq 0$. We can conclude that $\left\{Q_{n}\right\}_{n \geq 0}$ is symmetric. Using the Proposition 2, we obtain

$$
Q_{n}(x)=q^{-n} P_{n}(q x), \quad n \geq 0 .
$$

Then we also conclude that $\left\{P_{n}\right\}_{n \geq 0}$ is symmetric. Moreover, the relation (3.11) corresponds to a Pearson's equation of $q$-Hermite linear functional, hence $Q_{n}(x)$ is the $q$-Hermite polynomial. In addition, we have $Q_{n}(x)=q^{-n} P_{n}(q x), n \geq 0$, then $P_{n}(x)$ is the scaled $q$-Hermite polynomial.

\subsection{Orthogonal polynomials via raising operator $(X+1)-\lambda \tau_{-1}$}

In this part, we use the following lemma.

Lemma 4 [1]. The following properties hold

$$
\begin{gathered}
D_{w}(f g)(x)=f(x)\left(D_{w} g\right)(x)+g(x)\left(D_{w} f\right)(x)+w\left(D_{w} f\right)(x)\left(D_{w} g\right)(x), \quad f, g \in \mathcal{P} \\
D_{-w}(f u)=g\left(D_{-w} u\right)+\left(D_{-w} g\right)\left(\tau_{w} u\right), \quad f \in \mathcal{P}, \quad u \in \mathcal{P}^{\prime} \\
\tau_{b} \circ D_{w}=D_{w} \circ \tau_{b} \text { in } \mathcal{P} \text { and } \mathcal{P}^{\prime}, \quad b \in \mathbb{C}
\end{gathered}
$$

where

$$
D_{\omega} f(x):=\frac{\tau_{-\omega} f(x)-f(x)}{w}, \quad \omega \neq 0 \quad \text { and } \quad \tau_{-\omega} f(x)=f(x+\omega) .
$$


Recall the operator

$$
\begin{aligned}
\mathcal{S}_{\lambda}: \mathbb{P} & \longrightarrow \mathbb{P} \\
f & \longmapsto \mathcal{S}_{\lambda}(f)=(x+1)(f)-\lambda \tau_{-1} f .
\end{aligned}
$$

Definition 2. We call a sequence $\left\{P_{n}\right\}_{n \geq 0}$ of orthogonal polynomials $\mathcal{S}_{\lambda}$-classical if there exists a sequence $\left\{Q_{n}\right\}_{n \geq 0}$ of orthogonal polynomials such that $\mathcal{S}_{\lambda} P_{n}=Q_{n+1}, n \geq 0$.

For any MPS $\left\{P_{n}\right\}_{n \geq 0}$ we define the MPS $\left\{Q_{n}\right\}_{n \geq 0}$, given by

$$
Q_{n+1}(x):=\mathcal{S}_{\lambda} P_{n}(x), n \geq 0,
$$

or equivalently

$$
Q_{n+1}(x):=(x+1) P_{n}(x)-\lambda P_{n}(x+1), n \geq 0,
$$

with initial value $Q_{0}(x)=1$.

Our next goal is to describe all the $\mathcal{S}_{\lambda}$-classical polynomial sequences. Note that, we need $\lambda \neq 0$ to ensure that $\left\{Q_{n}\right\}_{n \geq 0}$ is an orthogonal sequence. Indeed, if we suppose that $\lambda=0$, the relation (3.13) becomes, for $x=-1, Q_{n+1}(-1)=0, n \geq 0$, which contradicts the orthogonality of $\left\{Q_{n}\right\}_{n \geq 0}$.

Clearly, the operator $\mathcal{S}_{\lambda}$ raises the degree of any polynomial. Such operator is called a raising operator $[9,13,19]$. By transposition of the operator $\mathcal{S}_{\lambda}$, we get

$$
{ }^{t} \mathcal{S}_{\lambda}=(X+1)-\lambda \tau_{1} .
$$

Denote by $\left\{u_{n}\right\}_{n \geq 0}$ and $\left\{v_{n}\right\}_{n \geq 0}$ the dual basis in $\mathbb{P}^{\prime}$ corresponding to $\left\{P_{n}\right\}_{n \geq 0}$ and $\left\{Q_{n}\right\}_{n \geq 0}$, respectively. Then, according to Lemma 1 and (3.14), the relation

$$
(x+1) v_{n+1}-\lambda \tau_{1} v_{n+1}=u_{n}, \quad n \geq 0,
$$

holds. Assume that $\left\{P_{n}\right\}_{n \geq 0}$ and $\left\{Q_{n}\right\}_{n \geq 0}$ are MOPS satisfying

$$
\begin{aligned}
& \left\{\begin{array}{l}
P_{0}(x)=1, \quad P_{1}(x)=x-\beta_{0}, \\
P_{n+2}(x)=\left(x-\beta_{n+1}\right) P_{n+1}(x)-\gamma_{n+1} P_{n}(x), \quad \gamma_{n+1} \neq 0, \quad n \geq 0,
\end{array}\right. \\
& \left\{\begin{array}{l}
Q_{0}(x)=1, \quad Q_{1}(x)=x-\rho_{0}, \\
Q_{n+2}(x)=\left(x-\rho_{n+1}\right) Q_{n+1}(x)-\varrho_{n+1} Q_{n}(x), \quad \varrho_{n+1} \neq 0, \quad n \geq 0 .
\end{array}\right.
\end{aligned}
$$

Next, a first result will be deduced as a consequence of the relations (3.13), (3.15) and (3.16).

Proposition 3. The sequences $\left\{P_{n}\right\}_{n \geq 0}$ and $\left\{Q_{n}\right\}_{n \geq 0}$ satisfy the following finite type relation

$$
Q_{n}(x)=\tau_{-1} P_{n}(x), \quad n \geq 0,
$$

with

$$
\begin{gathered}
\rho_{n+1}=\beta_{n}, \quad n \geq 0, \\
\varrho_{n+1}=\gamma_{n}+\lambda, \quad n \geq 0,
\end{gathered}
$$

and with the convention $\gamma_{0}=0$. 
P r o o f. Multiplying (3.15) by $x+1$, we obtain

$$
(x+1) P_{n+2}(x)=\left(x-\beta_{n+1}\right)(x+1) P_{n+1}(x)-\gamma_{n+1}(x+1) P_{n}(x), \quad n \geq 0 .
$$

Applying $\lambda \tau_{-1}$ to the (3.15) and taking the difference between the two resulting equations, we obtain

$$
\begin{gathered}
(x+1) P_{n+2}(x)-\lambda\left(\tau_{-1} P_{n+2}\right)(x)=\left(x-\beta_{n+1}\right)\left((x+1) P_{n+1}(x)-\lambda\left(\tau_{-1} P_{n+1}\right)(x)\right) \\
-\gamma_{n+1}\left((x+1) P_{n}(x)-\lambda\left(\tau_{-1} P_{n}\right)(x)\right)-\lambda P_{n+1}(x+1) .
\end{gathered}
$$

Substituting (3.13) in the last equation, we get

$$
Q_{n+3}(x)=\left(x-\beta_{n+1}\right) Q_{n+2}(x)-\gamma_{n+1} Q_{n+1}(x)-\lambda P_{n+1}(x+1), \quad n \geq 0 .
$$

Using the three-term recurrence relation (3.16), we get

$$
\lambda P_{n+1}(x+1)=\left(\rho_{n+2}-\beta_{n+1}\right) Q_{n+2}(x)+\left(\varrho_{n+2}-\gamma_{n+1}\right) Q_{n+1}(x), \quad n \geq 0 .
$$

In fact, this result is valid for $n+1$ replaced by $n$. Then, by comparing the degrees in the previous equation, we get $\rho_{n+1}=\beta_{n}$ and $\varrho_{n+1}=\gamma_{n}+\lambda, n \geq 0$, and $Q_{n}(x)=\tau_{-1} P_{n}(x), \quad n \geq 0$, with the convention $\gamma_{0}=0$.

The following result is a straightforward consequence of Proposition 3.

Lemma 5. The forms $u_{0}$ and $v_{0}$ satisfy the following relation

$$
\tau_{1} v_{0}=u_{0} .
$$

According to Lemma 5, and based on some characterizations of Charlier polynomials [1], we can state the following theorem.

Theorem 2. The Charlier polynomial sequence $\left\{C_{n}^{\lambda}(x)\right\}_{n \geq 0}$ where $\lambda>0$, is the only $\mathcal{S}_{\lambda^{-}}$ classical orthogonal sequence. More precisely, we have for $n \geq 0$ :

$$
\begin{gathered}
P_{n}(x)=C_{n}^{\lambda}(x), \\
Q_{n}(x)=C_{n}^{\lambda}(x+1) .
\end{gathered}
$$

P r o o f. Assume that $\left\{P_{n}\right\}_{n \geq 0}$ is a monic $\mathcal{S}_{\lambda}$-classical orthogonal sequence. Then there exists a monic orthogonal sequence $\left\{Q_{n}\right\}_{n \geq 0}$ satisfying (3.13), which gives by transposition the following system

$$
\left\langle v_{0},(x+1) P_{n}(x)-\lambda P_{n}(x+1)\right\rangle=\left\langle v_{0}, Q_{n+1}(x)\right\rangle=0, \quad n \geq 0 .
$$

But the left hand side reads as

$$
\left\langle(x+1) v_{0}-\lambda \tau_{1} v_{0}, P_{n}(x)\right\rangle=0, \quad n \geq 0 .
$$

In other words,

$$
(x+1) v_{0}-\lambda \tau_{1} v_{0}=0 .
$$

Applying the operator $\tau_{-1}$, we obtain

$$
(x+2) \tau_{-1} v_{0}-\lambda v_{0}=0 .
$$

Equivalently,

$$
(x+1) \tau_{-1} v_{0}+\tau_{-1} v_{0}-(x+1) v_{0}+(x+1) v_{0}-\lambda v_{0}=0,
$$


which also gives

$$
(x+1)\left[\tau_{-1} v_{0}-v_{0}\right]+\tau_{-1} v_{0}+(x+1) v_{0}-\lambda v_{0}=0,
$$

or equivalently

$$
(x+1) D_{1} v_{0}+\tau_{-1} v_{0}+(x+1) v_{0}-\lambda v_{0}=0 .
$$

By using Lemma 4, the last relation becomes

$$
D_{1}\left(x\left(\tau_{1} v_{0}\right)\right)+(x-\lambda)\left(\tau_{1} v_{0}\right)=0,
$$

which means that $v_{0}=\tau_{-1} C(\lambda)$, where $C(\lambda)$ is the Charlier form with $\lambda>0$. In addition, using the Proposition 3, we obtain that $P_{n}(x)=C_{n}^{\lambda}(x)$ are the monic Charlier polynomials and then

$$
Q_{n}(x)=C_{n}^{\lambda}(x+1), \quad n \geq 0 .
$$

\section{Conclusion and prospects}

We described Hahn's problem for some perturbed raising operators of the operator $X-c$ using the Pearson equation, which is satisfied by the corresponding linear functionals. Indeed, we have proved that the $q$-Hermite (resp. Charlier) polynomial is the only $H_{\alpha, q}$-classical (resp. $\mathcal{S}_{\lambda}$-classical) orthogonal polynomial, where $H_{\alpha, q}:=X+\alpha H_{q}$ and $\mathcal{S}_{\lambda}:=(X+1)-\lambda \tau_{-1}$.

Now, using (3.17), (3.18) and (3.12), we obtain

$$
\mathcal{S}_{\lambda} C_{n}^{\lambda}(x)=C_{n+1}^{\lambda}(x+1), \quad n \geq 0,
$$

which gives, by induction, the following formula

$$
\mathcal{S}_{\lambda}^{(m)} C_{n}^{\lambda}(x)=C_{n+m}^{\lambda}(x+m), \quad n \geq 0,
$$

where $\mathcal{S}_{\lambda}^{(m)}=\mathcal{S}_{\lambda}^{(m)} \circ \cdots \circ \mathcal{S}_{\lambda}^{(m)}$.

Making $n=0$ in (4.1) we get

$$
\mathcal{S}_{\lambda}^{(m)}(1)=C_{m}^{\lambda}(x+m), \quad m \geq 0 .
$$

For prospects, we can replace the operator $H_{q}$ in Subsection 3.1 by the Dunkl operator $\left(T_{\mu}:=D+2 \mu H_{-1}\right.$, see [6]) and study the same problem. Indeed, we have [6]

$$
\left(X-\frac{1}{2} T_{\mu}\right) H_{n}^{\mu}(x)=\frac{\gamma_{\mu}(n+1)}{2 \gamma_{\mu}(n)(n+1)} H_{n+1}^{\mu}(x), \quad n \geq 0,
$$

where $H_{n}^{\mu}(x)$ is the monic generalized Hermite polynomial and where $\gamma_{\mu}(n)$ is defined by

$$
\gamma_{\mu}(2 m)=\frac{2^{2 m} m ! \Gamma(m+\mu+1 / 2)}{\Gamma(\mu+1 / 2)}, \quad \text { and } \quad \gamma_{\mu}(2 m+1)=\frac{2^{2 m+1} m ! \Gamma(m+\mu+1 / 2)}{\Gamma(\mu+3 / 2)} .
$$

In view of (4.2), we can say that $\left\{H_{n}^{\mu}\right\}_{n \geq 0}$ is an $\mathcal{O}$-classical polynomial sequence, since it fulfills Hahn's property relatively to the raising operator

$$
\mathcal{O}:=X-\frac{1}{2} T_{\mu},
$$

i.e., it is an orthogonal polynomial sequence whose sequence of $\mathcal{O}$-derivatives is also orthogonal. 


\section{Acknowledgements}

The authors would like to thank the referees for their corrections and many valuable suggestions.

\section{REFERENCES}

1. Abdelkarim F., Maroni P. The $D_{\omega}$-classical orthogonal polynomials. Result. Math., 1997. Vol. 32, No. 12. P. 1-28. DOI: $10.1007 /$ BF03322520

2. Aloui B. Characterization of Laguerre polynomials as orthogonal polynomials connected by the Laguerre degree raising shift operator. Ramanujan J., 2018. Vol. 45, No. 2. P. 475-481. DOI: $10.1007 / \mathrm{s} 11139-017-9901-\mathrm{x}$

3. Aloui B. Chebyshev polynomials of the second kind via raising operator preserving the orthogonality. Period. Math. Hung., 2018. Vol. 76, No. 1. P. 126-132. DOI: 10.1007/s10998-017-0219-7

4. Aloui B., Khériji L. Connection formulas and representations of Laguerre polynomials in terms of the action of linear differential operators. Probl. Anal. Issues Anal., 2019. Vol. 8(26), No. 3. P. 24-37. DOI: $10.15393 / \mathrm{j} 3$. art.2019.6290

5. Area I., Godoy A., Ronveaux A., Zarzo A. Classical symmetric orthogonal polynomials of a discrete variable. Integral Transforms Spec. Funct., 2004. Vol. 15, No. 1. P. 1-12. DOI: $10.1080 / 10652460310001600672$

6. Ben Cheikh Y., Gaied M. Characterization of the Dunkl-classical symmetric orthogonal polynomials. Appl. Math. Comput., 2007. Vol. 187, No. 1. P. 105-114. DOI: 10.1016/j.amc.2006.08.108

7. Ben Salah I., Ghressi A., Khériji L. A characterization of symmetric $T_{\mu}$-classical monic orthogonal polynomials by a structure relation. Integral Transforms Spec. Funct., 2014. Vol. 25, No. 6. P. 423-432. DOI: $10.1080 / 10652469.2013 .870339$

8. Bouanani A., Khériji L., Tounsi M.I. Characterization of $q$-Dunkl Appell symmetric orthogonal $q$ polynomials. Expo. Math., 2010. Vol. 28, No. 4. P. 325-336. DOI: 10.1016/j.exmath.2010.03.003

9. Chaggara H. Operational rules and a generalized Hermite polynomials. J. Math. Anal. Appl., 2007. Vol. 332, No. 1. P. 11-21. DOI: 10.1016/j.jmaa.2006.09.068

10. Chihara T.S. An Introduction to Orthogonal Polynomials. New York: Gordon and Breach, 1978. 249 p.

11. Hahn W. Über die Jacobischen polynome und zwei verwandte polynomklassen. Math. Z., 1935. Vol. 39. P. 634-638.

12. Khériji L., Maroni P. The $H_{q}$-classical orthogonal polynomials. Acta. Appl. Math., 2002. Vol. 71, No. 1. P. 49-115. DOI: 10.1023/A:1014597619994

13. Koornwinder T.H. Lowering and raising operators for some special orthogonal polynomials. In: Jack, Hall-Littlewood and Macdonald Polynomials, V.B. Kuznetsov, S. Sahi (eds.). Contemp. Math., vol. 417, 2006. P. 227-239. DOI: $10.1090 / \mathrm{conm} / 417$

14. Maroni P., Mejri M. The $I_{(q, \omega)}$-classical orthogonal polynomials. Appl. Numer. Math., 2002. Vol. 43, No. 4. P. 423-458. DOI: 10.1016/S0168-9274(01)00180-5

15. Maroni P. Fonctions Eulériennes, Polynômes Orthogonaux Classiques. Techniques de l'Ingénieur, Traité Généralités (Sciences Fondamentales), 1994. Vol. 154 A. Paris. P. 1-30.

16. Maroni P. Une théorie algébrique des polynômes orthogonaux. Application aux polynômes orthogonaux semi-classiques. In: Orthogonal Polynomials and their Applications, C. Brezinski et al. (eds.). IMACS Ann. Comput. Appl. Math., vol. 9. Basel: Baltzer,1991. P. 95-130.

17. Maroni P. Variations autour des polynmes orthogonaux classiques. C. R. Acad. Sci. Paris Sér. I Math., 1991. Vol. 313. P. 209-212.

18. Sonine N. J. On the approximate computation of definite integrals and on the entire functions occurring there. Warsch. Univ. Izv., 1887. Vol. 18. P. 1-76.

19. Srivastava H. M., Ben Cheikh Y. Orthogonality of some polynomial sets via quasi-monomiality. Appl. Math. Comput., 2003. Vol. 141, No. 2-3. P. 415-425. DOI: 10.1016/S0096-3003(02)00961-X 